\title{
The Travel and Tropical Medicine Manual, 4th Edition
}

Jong E, Sanford C, editors. Saunders Elsevier, 2008. Soft cover, 688 pages. ISBN 978-1-4160-2613-6.

The Travel and Tropical Medicine Manual is a comprehensive reference that provides the clinician with the fundamentals of travel and tropical medicine. As international travel becomes more commonplace, health care providers need to be informed about the prevention and treatment of communicable diseases and must also know how to counsel their patients on travelrelated health issues.

In this manual, 49 contributors cover a broad range of topics related to travel and tropical medicine. The 45 chapters are

JCPH - Vol. 62, n $n^{\circ} 5$ - septembre-octobre 2009 
divided into 7 main sections (Pre-Travel Advice, Advice for Special Travelers, Fever, Diarrhea, Skin Lesions, Sexually Transmitted Diseases, and Worms). Each section is colour-coded, and the content is well-organized, succinct, and effectively highlighted by illustrative figures, tables, and images. The compact size makes this an ideal pocket reference, although the font size of the text could have been larger.

The manual provides practical and instructive information for counselling travellers before they leave home. This includes details on preventing communicable diseases, as well as discussion of travel health issues such as time-zone changes, air pollution, altitude illness, and diving medicine. In addition, comprehensive guidance for the treatment of post-travel conditions is provided. Some key chapters are those covering vaccinations, malaria prevention, traveller's diarrhea (prevention and self-treatment), HIV infection, tropical dermatology, and sexually transmitted infections.

Each chapter begins with a concise preamble and background for the topic. In sections where an infectious process is the main focus, a description of the pathogenesis and disease is provided, including the history and clinical manifestations required to make a diagnosis. The content uses medical terminology intended for health care providers. Pertinent material is effectively summarized in tables, and colourful maps are used to highlight the prevalence of specific diseases.

The pharmacological management of infections (drug, dose, duration of treatment, and common adverse effects) is generally summarized in tables. Details of the drugs are also given in the text, but in brief, general terms. For more comprehensive drug information, readers may need to refer to other resources. A useful feature is the list of reference articles provided at the end of each chapter.

Other practical information in the manual includes a table on the use of melatonin to prevent jet lag and another on potential drug interactions between HIV antiretroviral therapy and travel-related medications. In addition, there is a list of online resources where readers can find up-to-date travel information.

In summary, The Travel and Tropical Medicine Manual is a good reference for clinicians who wish to review the concepts of travel and tropical medicine and those who require a resource for specific clinical indications. The target audience consists of infectious disease physicians, primary care physicians, and other health care practitioners who specialize in travel medicine. Pharmacists who wish to broaden their knowledge in travel medicine and those who are avid world travellers would benefit from this book.

Tim T Y Lau, PharmD, ACPR, FCSHP

Pharmacotherapeutic Specialist in Infectious Diseases

Vancouver General Hospital

Clinical Associate Professor

Faculty of Pharmaceutical Sciences

University of British Columbia

Vancouver, British Columbia 